\section{Spontane Heilung eines pulmonalen Aspergilloms: „Actinomyces zerstört Aspergillus“}

J. Hartmann, R. Keller

Klinik Barmelweid, Aarau/Schweiz
Zusammenfassung: Bei einem 79-jährigen Patienten hatte sich im Anschluss an eine abszedierende Infarktpneumonie ein riesiges Aspergillom im kavernös veränderten rechten Oberlappen entwickelt. Nach kompliziertem Verlauf über 2 Jahre mit anämisierender Hämoptoe sowie kontinuierlicher Größenzunahme des Myozetoms entwickelte sich eine akute eitrige Gangrän des zerstörten Oberlappens mit Nachweis von Staphylococcus aureus und Actinomyces israelii, hingegen keine Aspergillen mehr in perkutan aspirierten Proben. Nach transthorakaler Spüldrainage der Abszesshöhle während 30 Tagen war das Aspergillom auch radiologisch nicht mehr nachweisbar, lediglich die Präzipitine blieben noch deutlich positiv. Im weiteren mehrmonatigen Verlauf waren keine pulmonalen Komplikationen mehr zu verzeichnen und der Patient verstarb schließlich an einem rupturierten Aortenaneurysma. Es wird vermutet, dass die spontane Lyse des riesigen Aspergilloms auf die infizierte Lungengangrän zurückzuführen ist, wodurch dem Myozetom die nutritive Grundlage entzogen wurde. Ein ähnlicher Mechanismus ist möglicherweise auch für die allerdings zahlenmäßig bisher bescheidenen Erfolge bei perkutaner Injektion von amphotericinhaltiger Paste verantwortlich, wie dies in der neueren Literatur für inoperable Aspergillome empfohlen wird.

Spontaneous Lysis of Pulmonary Aspergilloma: Aspergillus Destroyed by Actinomyces: A 79 years old patient developed a large pulmonary aspergilloma in the cavities of his right upper lobe after postinfarctional pneumonia with local abscess formation. The clinical follow-up was characterized by recurrent hemoptysis resulting in marked anemia as well as by a continuous growth of the mycetoma. Suddenly a purulent gangrene of the whole upper lobe occured infected by actinomyces israelii and staphylococcus but not aspergillus as it could be demonstrated in specimens from repeated thransthoracic needle aspirations. After percutaneously inserted chest-tube drainage during $\mathbf{3 0}$ days the upper lobe cavity cleared up and the previously impressive aspergilloma had disappeared completely, however, the serum precipitins from aspergillus fumigatus still remained positive. After a course of several months without further pulmonary complications the patient finally died from a ruptured aortic aneurysm. It is suggested, that the spontaneous lysis of the aspergilloma was due to a deprivation of it' nutritive basis by the infected pulmonary tissue. A similar mechanism may also account for a sometimes successfull treat-

Pneumologie 2000; 54: $392-394$

(c) Georg Thieme Verlag Stuttgart · New York ISSN 0934-8387 ment of pulmonary aspergilloma after injection of an amphotericin containing paste as a novel therapeutic strategy which is recommended in the case of patient's inoperable conditions.

\section{Einleitung}

Das Aspergillom ist die häufigste pulmonale Manifestationsform einer durch Aspergillen verursachten Erkrankung beim Menschen. Generell kann sich ein Aspergillom lediglich bei vorbestehenden pulmonalen Missbildungen entwickeln, so insbesondere in Kavernen oder Zysten, wie man sie bei chronischer Tuberkulose, Sarkoidose oder zystischer Bronchiektasie antrifft. Lediglich mit der chirurgischen Entfernung und Sanierung der bronchopulmonalen Malformationen kann eine definitive Heilung des pulmonalen Aspergilloms erzielt werden. Indessen ist die chirurgische Sanierung, insbesondere bei älteren Patienten und vorbestehender disseminierter Lungenerkrankung, oft risikoreich oder sogar kontraindiziert. Die medikamentöse antimykotische Therapie ist in diesen Fällen zumeist wirkungslos, kostspielig und mit erheblichen Nebenwirkungen verbunden. Gelegentlich kommt es allerdings auch zur spontanen Lyse des Aspergilloms, wie dies im nachfolgenden Fall eines Patienten mit inoperablem Aspergillom beschrieben wird.

\section{Anamnese}

Im Januar 1997 erlitt der damals 79-jährige Patient rezidivierend Lungenembolien mit anschließender abszedierender Infarktpneumonie im rechten Oberlappen. Trotz antibiotischer Therapie verblieb ein chronisches pneumonisches Infiltrat mit einem mehrkammerigen Kavernensystem. Wenige Monate später erfolgte eine erneute notfallmäßige Zuweisung wegen rezidivierender Hämoptoe. Radiologisch zeigte sich jetzt im Bereich des ursprünglich kavernös veränderten rechten Oberlappens eine homogene kugelige Raumfordung von $7 \times 5 \mathrm{~cm}$ Durchmesser mit schmalem begrenzenden Luftsaum, aufsitzend auf dem oberen rechten Hiluspol (Abb.1). Die Bronchoskopie ergab eine eitrige Sekretdrainage aus dem rechten Oberlappenbronchus mit Nachweis von Aspergillen im Grampräparat und in der Kultur, serologisch hohe Titer auf Präzipitine gegenüber Aspergillus fumigatus. Die Befunde waren somit charakteristisch für ein pulmonales Aspergillom. Auf eine chirurgische Sanierung wurde wegen erhöhtem operativen Risiko verzichtet und stattdessen eine perorale Therapie mit Itraconazol eingeleitet, nach 8-monatiger Therapie jedoch wegen fehlender radiologischer Rückbildung des 


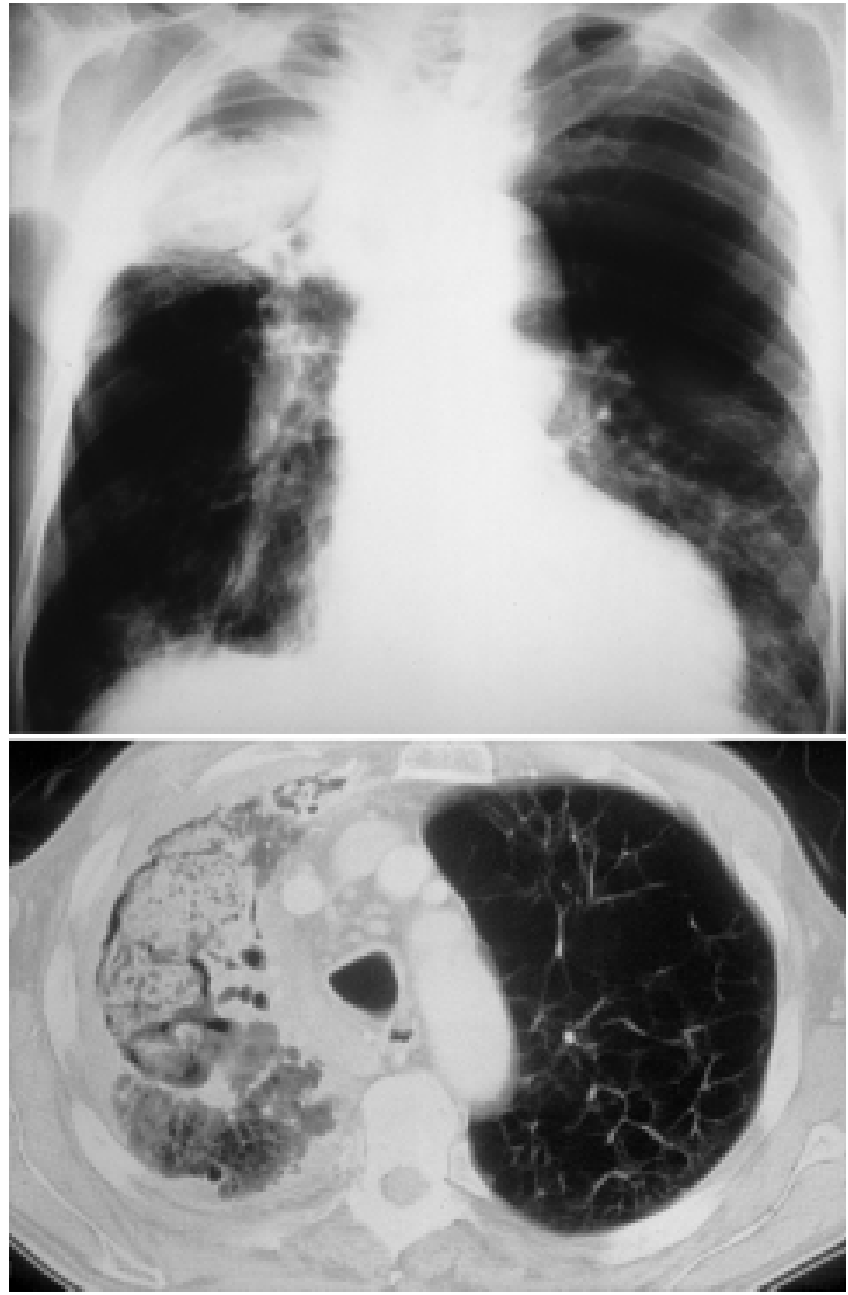

Abb. 1 79-jähriger Patient mit großem pulmonalen Aspergillom im kavernös veränderten rechten Oberlappen nach abszedierender Infarktpneumonie.

Aspergilloms wieder abgesetzt. Im Frühjahr 1999 erneute notfallmäßige Hospitalisation wegen akuter Verschlechterung des Allgemeinzustandes, Status febrilis, erneute Hämoptoe und massiven eitrigen Expektorationen.

\section{Befunde}

81-jähriger Patient in stark reduziertem Allgemein- und Ernährungszustand (Gewicht 76,5 kg, Größe $183 \mathrm{~cm}$, BodymassIndex $22,8 \mathrm{~kg} / \mathrm{m}^{2}$ ), klares Bewusstsein und allseits orientiert, kardial kompensiert, Herzfrequenz 100/min, Blutdruck 145/ 75 mm Hg, Tachypnoe, 28/min, Dämpfung und abgeschwächtes Atemgeräusch über dem rechten Oberfeld, fehlende Fußpulse beidseits. Labor: Hämoglobin $121 \mathrm{~g} / \mathrm{L}$, Leukozyten 14,1 g/L (11\% Stabkernige, 77\% Segmentkernige, 2\% Eosinophile, 10\% Lymphozyten, Thrombozyten 300000 ), Elektrolyte, Kreatinin und Transaminasen normal, C-reaktives Protein stark erhöht $(240 \mathrm{mg} / \mathrm{L})$ Blutgase: $\mathrm{pO}_{2} 46,1 \mathrm{mmHg}, \quad \mathrm{pCo}_{2}$ $34,1 \mathrm{~mm} \mathrm{Hg}, \mathrm{pH} 7,49, \mathrm{SaO}_{2} 76 \%$. Im Thoraxröntgenbild eine homogendichte Verschattung des gesamten rechten Oberfeldes, mit Spiegelbildung in der Lungenspitze, außerdem disseminierte feinfleckige Herdbildungen in beiden Unterfeldern (Abb. 2).

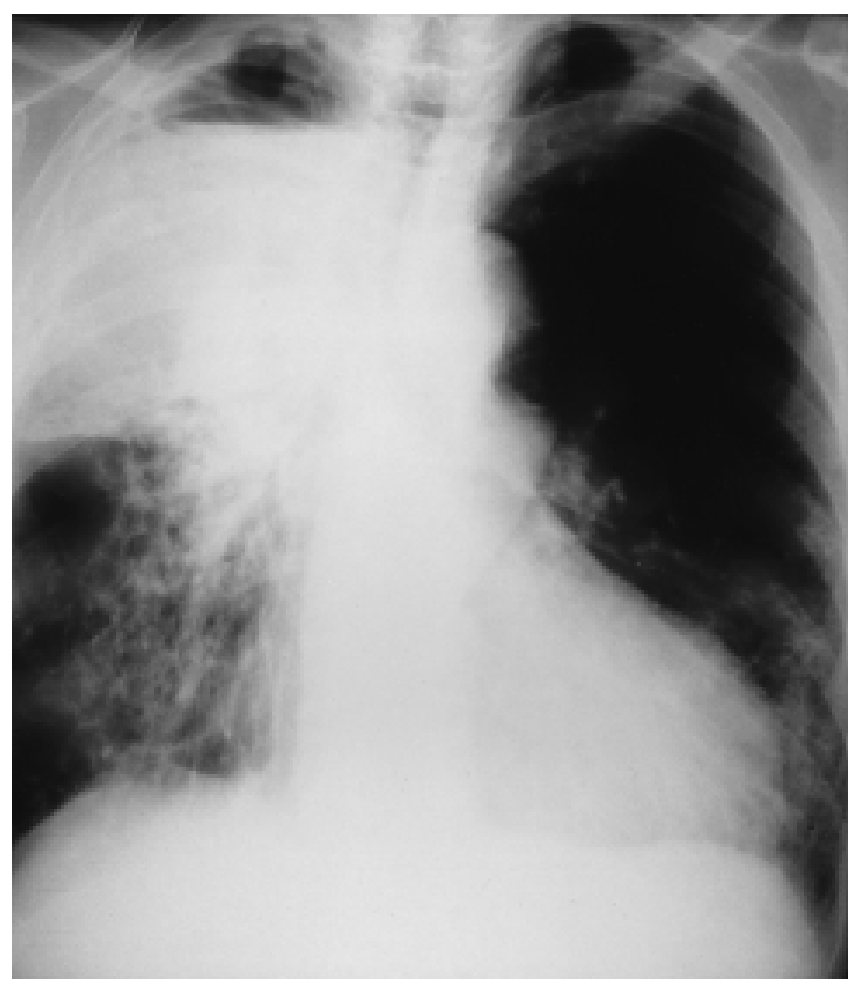

Abb. 2 Eitrige Lungengangrän mit Spiegelbildung des rechten Oberlappens.

\section{Differentialdiagnose}

1. Sekundäre Pneumonie des kavernös veränderten rechten Oberlappens

2. Aspergillom des rechten Oberlappens

3. Disseminierte Bronchopneumonien des rechten und linken Unterlappens.

\section{Verlauf}

Unverzügliche Therapie mit Breitbandantibiotika (Amoxiclavonat $3 \times 1 \mathrm{~g}$ täglich); trotzdem weiterhin quälender Husten mit massiven eitrigen Expektorationen, wodurch sich der betagte Patient physisch zunehmend erschöpfte. Es erfolgte die perkutane Punktion des rechten Oberlappens mit Aspiration von eitrigem Material und bakteriologisch Nachweis von Staphylococcus aureus und Actinomyces israelii, hingegen keine Aspergillen. Unter der Annahme eines gangränösen nekrotischen Zerfalls des rechten Oberlappens wurde transthorakal eine Spüldrainage parascapulär von dorsal her eingelegt, worauf sich ca. 1 Liter einer rahmig-eitrigen Flüssigkeit entleerte. Auch in diesem Material konnte kulturell Actinomyces israelii, hingegen erstaunlicherweise kein Aspergillus fumigatus mehr nachgewiesen werden. Die Spüldrainage wurde während insgesamt 30 Tagen belassen mit täglicher Instillation von $250 \mathrm{ml}$ physiologischer Kochsalzlösung. Der Allgemeinzustand des Patienten verbesserte sich dabei von Tag zu Tag, die purulenten Expektorationen bildeten sich eklatant zurück, offensichtlich seit dem die eitrige Flüssigkeit nun durch die Drainage über ein Bülau-System nach außen entweichen konnte. Das initial auf $241 \mathrm{mg} / \mathrm{L}$ stark erhöhte Creaktive Protein normalisierte sich auf $8 \mathrm{mg} / \mathrm{L}$. Im Thoraxüber- 
sichtsbild war jetzt anstelle des rechten Oberlappens eine septierte große Höhle getreten mit verdicktem basalen Randwall, weiterhin persistierten die disseminierten fleckigen und konfluierenden Infiltrationen vor allem im rechten Unterfeld. Die ursprünglich kugelige und als Aspergillom identifizierte Raumforderung war verschwunden (Abb. 3).

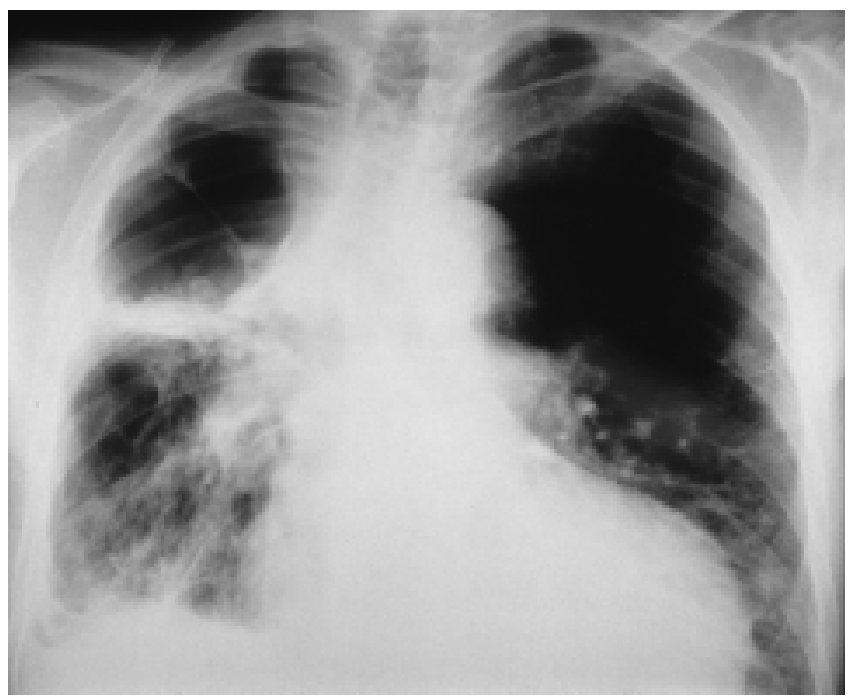

Abb. 3 Gereinigte Höhle im rechten Oberlappenbereich nach perthorakaler Spüldrainage und spontaner Lyse des Aspergilloms.

Beurteilung: Gangrän des rechten Oberlappens

- Infektion durch Actinomyces israelii und Staphylococcus aureus

- lytischer Zerfall des vorbestehenden Aspergilloms

\section{Diskussion}

Bemerkenswert an diesem Fallbericht ist die spontane und vollständige Lyse eines riesigen Aspergilloms des rechten Oberlappens im Anschluss an eine Infektion des vorbestehenden Kavernensystems mit Staphylococcus aureus und Actinomyces israelii. Nachdem im vorgängigen und mehr als 2jährigen Verlauf eine stetige Größenzunahme des Aspergilloms zu verzeichnen war, muss davon ausgegangen werden, dass die bakterielle Superinfektion die Lyse des Mycetoms direkt verursacht oder zumindest gefördert hat. In der Literatur werden tatsächlich gelegentlich spontane Lysen eines Aspergilloms beschrieben, so beispielsweise in einer Studie von Singh [1], wo bei 49 Patienten mit pulmonalem Aspergillom über einen Zeitraum von 6 Jahren tatsächlich in einem Fall eine spontane Lyse beobachtet wurde. In einer anderen Arbeit von Garros [2] wurde anhand retrospektiver Untersuchung bei 31 Patienten sogar in 4 Fällen eine spontane Lyse festgestellt; allerdings ohne dass hierbei besondere Begleitkomplikationen beschrieben wurden.

Grundsätzlich ist die Therapie der Wahl bei pulmonalem Aspergillom nach wie vor die chirurgische Entfernung des Mycetoms und auch der geschädigten und zumeist kavernös veränderten Lungenstrukturen, welche ja in der Regel die Voraussetzungen für das Einnisten des Mycetoms bilden. In einer Verlaufsstudie von el Oakley [3] wurde das Ergebnis der chirurgischen Entfernung des Aspergilloms retrospektiv untersucht und es konnte gezeigt werden, dass von 24 Patienten nach einer mittleren Beobachtungszeit von 17 Monaten noch 19 am Leben und symptomfrei waren. $\mathrm{Zu}$ einem ähnlichen Schluss kommt eine Studie von Chen u. Mitarb. [4], in welcher 64 Patienten retrospektiv über 28 Jahre untersucht wurden. Bei inoperablen Patienten sind die therapeutischen Möglichkeiten indessen nach wie vor beschränkt und die Prognose entsprechend unsicher. Die parenterale Verabreichung von Amphotericin B oder neuerdings die perorale Therapie mit Itraconazol bewirkt zwar gelegentlich eine Verkleinerung des Aspergilloms; eine Beseitigung oder Abheilung ist bislang damit aber in keinem einzigen Fall erzielt worden [5]. Etwas erfolgversprechender scheinen Behandlungstechniken, bei welchen das Antimykotikum direkt in die Aspergillomhöhle appliziert wird. In einer Studie von Giron u. Mitarb. [6] wurde bei 40 Patienten eine amphotericinhaltige Paste mittels CTgesteuerter perkutaner Injektion in die Aspergillomhöhle eingebracht. Allerdings konnte lediglich bei 3 Patienten innerhalb einer Verlaufsbeobachtung von 6-28 Monaten das Aspergillom damit vollständig beseitigt werden.

Zur Zeit ist die konservative Behandlung des pulmonalen Aspergilloms immer noch unbefriedigend. Die Therapie der Wahl ist weiterhin die chirurgische Entfernung des Mycetoms und Sanierung der Defekte. In inoperablen Situationen verspricht die lokale perkutane Injektion von Amphotericin B eine prozentual allerdings eher bescheidene Heilungschance. Möglicherweise ist der Behandlungserfolg mit lokalen Maßnahmen nicht nur durch das Antimykotikum allein, sondern durch das mit dem Einführen der Paste verbundene anaerobe Milieu verantwortlich, wodurch dem Mycetom die nutritive Grundlage entzogen wird. Dies könnte beispielsweise auch in dem von uns geschilderten Fall geschehen sein, wo sich die Aspergillenhöhle in eine eitrige Gangrän umgewandelt hatte.

\section{Literatur}

${ }^{1}$ Singh P, Kumar P, Bhagi RP et al. Pulmonary aspergillomaradiological observations. Indian J Chest Dis Allied Sci 1989; 31: 177

${ }^{2}$ Garros GJ, Ruiz GE, Vara QF et al. Pulmonary aspergillomas. Analysis of 31 patients. Arch Bronconeumol 1994; 30: 424

${ }^{3}$ el Oakley R, Petrou M, Goldstraw P. Indications and outcome of surgery for pulmonary aspergilloma. Thorax 1997; 52: 813

${ }^{4}$ Chen JC, Chang YL, Luh SP. Surgical treatment for pulmonary aspergilloma: a 28 year experiance. Thorax 1997; 9: 810

${ }^{5}$ Wierzbicka M, Wesolowski S, Podsiadlo B et al. Treatment of patients with pulmonary aspergilloma with itraconazole. Pneumonol Allergol Pol 1996; 64: 59

${ }^{6}$ Giron J, Poey C, Fajadet P et al. CT-guided percutaneous treatment of inoperable pulmonary aspergillomas: a study of 40 cases. Eur J Radiol 1998; 28: 235

Prof. Dr. med. R. Keller

Klinik Barmelweid

5017 Barmelweid/Aarau

Schweiz 